\title{
Le numérique pour la pédagogie en sciences de la santé : intégrer les technologies pour améliorer nos pratiques
}

\author{
Digital technology for pedagogy in health sciences: New technologies for better \\ practices
}

\author{
Numérique, digital, informatique : de quoi \\ parle-ton?
}

Il est parfois difficile de s'y retrouver dans les termes utilisés pour faire référence aux technologies de l'information et de la communication. Le dictionnaire Larousse définit le terme «numérique» comme quelque chose «qui relève des nombres; qui se fait avec des nombres, ou qui est représenté par un nombre». Le terme «digital» est l'équivalent anglais. Sa racine est latine: digitalis («qui a l'épaisseur d'un doigt»), dérivé de digitus («doigt»). Le terme anglais «digit» fait donc référence au fait que l'on compte sur ses doigts (de 1 à 10) et digital à tout ce qui utilise les nombres. Les deux mots sont souvent utilisés pour désigner tout ce qui est relié, de près ou de loin, à l'univers informatique.

L'utilisation que l'on en fait évolue et fluctue selon les auteurs et selon les années. Selon Anthony Mathé, docteur en sciences du langage et en sciences de la communication à l'École des hautes études en sciences de l'information et de la communication (Celsa-Sorbonne) et à l'Université de Paris 5, digital serait plutôt applicable à l'usager dans son expérience d'une technologie numérique, le «digital» faisant référence à ce qui se passe de l'autre côté de l'écran. Un smartphone serait ainsi à considérer comme un dispositif digital alors que la technologie qu'il emploie est numérique.

Évoquer ces différents termes (numérique, digital ou encore informatique) n'est aujourd'hui plus possible sans faire référence à Internet. Suite à l'apparition du premier protocole de communication qui lui donne naissance en 1969, son arrivée en Europe en 1985, et l'officialisation du terme «World Wide Web» en 1993, le web a contribué grandement à l'essor du numérique et de ses nouveaux usages.

Le numérique, de même que la définition que l'on en a et les usages qui s'y rattachent, sont donc en perpétuelle évolution depuis des années. On y fait souvent référence en parlant «d'innovation». Est-ce à juste titre?

\section{La perception du numérique et de l'innovation: une affaire de générations}

C'est une affaire de générations : chacun d'entre nous, pédagogues ou apprenants, a pris le train en marche et ce qui paraît innovant pour certains ne l'est plus tout à fait pour d'autres. Les sociologues font référence à quatre types de générations dans la société du $20^{\mathrm{e}}$ siècle: les babyboomers (nés entre 1946 et 1965), la génération $X$ (personnes nées entre 1965 et 1980), la génération Y (celles nées entre 1980 et 2000) et enfin la Z pour les individus nés à partir de l'an 2000. Les individus de la génération Y (et davantage encore ceux de la $\mathrm{Z}$ ) sont des «digital natives» : ils sont nés et ont grandi dans un environnement où le numérique domine le quotidien. Ils ne sont plus seulement consommateurs de contenu numérique. Ils sont des acteurs d'un web devenu social (le web 2.0 et ses réseaux sociaux). C'est un point essentiel à garder en tête lorsqu'on réfléchit à l'utilisation du numérique pour la pédagogie.

\section{Le numérique: une vague sur laquelle nous devons avancer}

L'arrivée d'internet, et avec lui des médias sociaux, a bouleversé notre mode d'accès à l'information. N'importe qui peut accéder instantanément à un savoir construit collectivement. La verticalité de l'acte pédagogique (de l'enseignant à l'apprenant) est abolie au profit d'échanges horizontaux. L'apprenant est au contact d'informations multiples qui évoluent en temps réel et qui constituent un savoir construit par des pairs.

Wikipedia en est sans doute l'exemple le plus emblématique. Cette encyclopédie en ligne, gratuite, compte aujourd'hui presque six millions d'articles (en anglais) construits par des communautés virtuelles d'individus. En 2005, une publication de la revue Nature faisait la démonstration que le contenu de cette encyclopédie était aussi fiable que celui de l'Encyclopæedia 
Britannica [1]. En 2012, Casebourne et al. comparaient la justesse des informations mais aussi la qualité, le style, les références et les jugements de Wikipedia (évalués par des experts) avec des entrées analogues d'autres encyclopédies et arrivaient à la conclusion que Wikipedia faisait mieux à tous points de vue pour les contenus en anglais en sciences médicales [2]. Un tel savoir est donc effectivement accessible à tous mais comme l'écrivait en 2012 Philippe Meirieu (professeur à l'Université Lumière-Lyon 2), «imaginer qu'Internet ouvre la porte au savoir, c'est ignorer ce qu'est savoir» en rappelant que le savoir est porté par une exigence de rigueur, de justesse et de vérité qui doit se forger dans le rapport au savoir médiatisé par l'enseignant [3]. En effet, savoir ne se résume pas à détenir une connaissance. Il est indispensable d'apprendre à l'interpréter, la contextualiser, l'utiliser et parfois la remettre en doute pour arriver à une notion de compétence. C'est bien sur là que l'enseignant a tout son rôle.

En tant que pédagogues, nous devons tirer parti de ces bouleversements et nous investir dans la construction de ce savoir construit collectivement. L'interactivité offerte par les réseaux sociaux le permet. Un éditorial récent de Pédagogie Médicale évoquait ainsi le potentiel de Twitter ${ }^{\circledR}$ en matière de formation tout au long de la vie dans le domaine du médicament [4].

\section{Un tsunami numérique en pédagogie?}

Emmanuel Davidenkoff, dans un livre publié en 2014 [5], décrivait le numérique comme une déferlante susceptible de bouleverser l'écosystème de l'éducation. À cette époque, l'engouement pour les MOOC (Massive Open Online Courses), présentés par les universités américaines comme une révolution pédagogique, en était un signe annonciateur. Ces formations de masse s'attaquaient au modèle économique et social de l'éducation: plus aucun étudiant (pauvre et éloigné des États-Unis) ne serait exclu des meilleures universités.

En France, la plateforme France Université Numérique (FUN MOOC) (https://www.fun-mooc.fr/) s'est investie dans ce nouveau modèle pédagogique en proposant «L'excellence de l'enseignement supérieur pour des cours en ligne, gratuits et ouverts à tous». Quelques années plus tard, les avis sont mitigés. En 2017, Le Monde dressait même un bilan très pessimiste en titrant «les MOOC font pschitt» [6]. Plusieurs obstacles ont été identifiés: leur mise en place et leur exploitation est extrêmement coûteuse en temps; leur aspect peu contraignant, gratuit et leur caractère asynchrone nuisent à la complétion du cours par l'apprenant. Les MOOC sont sans doute aujourd'hui un outil de formation tout au long de la vie mieux adapté aux contraintes d'actifs-apprenants qu'un cours en présentiel mais ils n'ont rien révolutionné en pédagogie. Ils se sont même très bien accommodés de sa forme la plus traditionnelle et conservatrice: celle du cours transmissif, dépourvu ou presque d'interaction (sauf par l'intermédiaire des forums de cours). Ces formations de masse, bien qu'elles puissent encore susciter un certain engouement, n'ont pas leur place dans nos maquettes formations initiales si l'on cherche à renforcer l'interactivité.

Dans nos universités, le numérique a permis le développement de l'enseignement à distance (EAD) sans que nous y soyons forcément préparés ou accompagnés. Les outils d'EAD sont multiples allant de simples outils d'édition permettant de créer des contenus à destination des étudiants (vidéos, questionnaires en ligne, etc.) à une multitude d'outils internet (forums, wiki, dossiers partagés, portfolio, etc.). L'ensemble se retrouve rassemblé dans des systèmes de gestion de contenu dédiés à l'apprentissage (en anglais 《Learning Management Systems» [LMS]). Les LMS, dont le plus connu est Moodle, sont de véritables piliers de l'EAD. Leur ergonomie est conçue pour que les apprenants se les approprient facilement (ce qui ne pose pas de problème aux générations $\mathrm{Y}$ ou $\mathrm{Z}$ ). En revanche, les administrer nécessite de bonnes connaissances informatiques ou une formation.

Nous devons nous approprier ces outils mais surtout réfléchir en amont de façon critique à leur pertinence et à la façon d'adapter notre pédagogie pour en tirer tout le bénéfice. L'EAD ne se limite pas à une approche technologique visant à reproduire les modalités de l'enseignement présentiel [7,8]. Il est aujourd'hui difficile d'affirmer que les apprenants bénéficiant de l'EAD soient pleinement aptes à tirer profit des avantages de cette modalité d'enseignement et d'apprentissage. La flexibilité, souvent présentée comme un atout de l'EAD, n'est pas forcément perçue comme telle par les apprenants qui peuvent avoir besoin de repères (notamment temporels) pour se mobiliser efficacement sur les taches d'apprentissage.

$\mathrm{Au}$-delà de l'EAD, le numérique a eu un impact sur nos pratiques en matière d'évaluation. L'apparition des machines permettant la correction automatisée des QCM a amené à les généraliser, par exemple en allant jusqu'à les proposer, en France, à l'examen terminal des études de médecine (épreuves classantes nationales informatisées).

\section{Le groupe « Numérique pour la pédagogie » de la Société internationale francophone d'éducation médicale (SIFEM)}

La question de savoir si nous devons utiliser ou non le numérique dans notre pédagogie n'est plus pertinente. Le numérique a d'ores et déjà envahit nos vies et il a modifié notre rapport aux savoirs et aux connaissances (en particulier pour les dernières générations de nos apprenants). Les technologies permettant de tirer parti du numérique existent. Cependant, de nombreuses questions se posent encore quant à la façon la plus pertinente de les utiliser et quant à leurs bénéfices pédagogiques. 
Dans ce contexte, la SIFEM a souhaité créer un groupe thématique dédié à l'intégration réussie du numérique en pédagogie. Ce groupe se donne pour mission principale de mener une réflexion sur les usages du numérique dans la formation en santé en travaillant sur une typologie. Les évolutions dans ce domaine étant constantes, cette typologie se devra d'être dynamique. Elle devra également intégrer une dimension analytique destinée à objectiver l'intérêt des dispositifs pour favoriser la dissémination des approches ou des outils les plus pertinents. À moyen terme, le groupe pourrait proposer d'accompagner les membres de la SIFEM souhaitant s'investir dans des approches de recherche ou d'évaluation sur le numérique en pédagogie.

Nicolas PICARD

Responsable du groupe thématique «Numérique pour la pédagogie » de la SIFEM*,

Faculté de pharmacie, Université de Limoges, Campus Marcland, 2 rue du docteur Marcland, 87025 Limoges,

France

*Mailto: Nicolas.picard@unilim.fr

\section{Références}

1. Giles J. Internet encyclopaedias go head to head. Nature 2005;438:900-1.

2. Casebourne I, Davies C, Fernandes M, Norman N. Assessing the accuracy and quality of Wikipedia entries compared to popular online encyclopedias. Brighton: Epic, 2012. [Online] : disponible sur : https://commons.wikimedia.org/wiki/ File:EPIC Oxford report.pdf.

3. Meirieu P. La pédāgogie et le numérique: des outils pour trancher? 2012. [On-line]: disponible sur: https://www. meirieu.com/ARTICLES/pedagogie_numerique.pdf.

4. Chouchana L, Auffret M, Geniaux H. Twitter , un outil de formation médicale continue? Pédagogie Médicale 2018: 19:91-3.

5. Davidenkoff E. Le tsunami numérique. Paris: Stock, 2014.

6. Miller M. Les MOOCS font pschitt. Le Monde 2017. [Online] : disponible sur : https://www.lemonde.fr/idees/article/ 2017/10/22/les-mooc-font-pschitt_5204379_3232.html.

7. Denef JF. Les nouvelles technologies de l'information et de la communication: quel impact auprès de nos étudiants? Pédagogie Médicale 2005;6:69-70.

8. Pelaccia T. Comment (mieux) former et évaluer les étudiants en médecine et en sciences de la santé? Louvain-la-Neuve: De Boeck Superieur, 2016. 